\title{
ENERGY TRANSFER THROUGH CONJUGATED POLYENES
}

ST. MAIER, H. PORT and H.C. WOLF

3. Physikalisches Institut der Universitāt, 7000 Stuttgart 80, (F. R. Germany)

F. EFFENBERGER and H. SCHLOSSER

Institut für Organische Chemie der Universität, 7000 Stuttgart 80 , (F. R. Germany)

\begin{abstract}
From an analysis of absorption, fluorescence and excitation spectra of linear polyene molecules with 4 to 13 double bonds and one or two aromatic substituents at the chain ends we conclude that the aromatic substituents can be excited individually, and that intramolecular energy transfer from one end group via the polyene to the other end group is possible.
\end{abstract}

\section{INTRODUCTION}

In the last years, increasing attention has been directed to the synthesis of organic molecules which are suitable for the transport and storage of information on a molecular basis. In this context, it is of high interest to find molecules in which energy absorbed at one end group of the molecule is transmitted intramolecularly to a different group at the opposite and. So the middle part of the molecule is a molecular wire for electronic excitation energy.

We have investigated the optical properties of polyenes with $4,5,8,9,12$ and 13 double bonds $\left(P_{n}\right)$ containing the substituents anthryl (A), naphthyl (N) and tetraphenylporphyrin (TPP) at one or both ends [1], Fig. 1.

First of all, we want to find out to which degree the electronic levels of the three constituents of the molecule (polyene and two substituents) are amalgamated into levels common to the whole molecule, and how this amalgamation is influenced by steric conditions. For this purpose, we have investigated absorption, emission and excitation spectra of the moleculea. Furtheron, it is most interesting to $f$ ind out whether an intramolecular energy transfer from one substituent (donor) to the second one (acceptor) mediated by the polyene chain can be identified. 


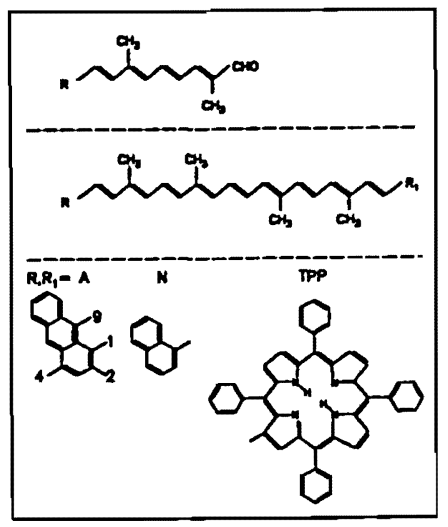

Fig. 1: Molecular Structures of $R-P_{4}-C H O, R-P_{9}-R_{1}$ and of the substituents $R, R_{1}$ : $A, \mathbf{N}$ and TPP

\section{EXPERIMENTAL}

Absorption spectra were recorded using a conventional spectrophotometer SP8-20 (Pye-Unicam). For excitaion of the fluorescence spectra, we used the continuum of a Xenon high pressure arc (Osram Suprasil $450 \mathrm{w}$ ) in combination with a $0,25 \mathrm{~m}$ monochromator (Jarrell-Ash), or for selective excitation at 514,5 or $257 \mathrm{~nm}$ an Argon Ion laser CR8 (Coherent). The emission spectra were recorded using a $1 \mathrm{~m}$ Jarrell-Ash double monochromator and a photomultiplier (EMI 9658), and corrected for spectral sensitivity.

Absorption spectra were measured at room temperature in solution of $\mathrm{CH}_{2} \mathrm{Cl}_{2}$, methylenchloride, at concentrations between $10^{-7}$ and $10^{-5} \mathrm{~mol} / 1$. In this range, all results are independent of concentration. Fluorescence spectra were taken in $\mathrm{n}$-hexane at $180 \mathrm{~K}$, in order to avoid photochemical decomposition which is non negligible at room temperature under strong illumination. By using low concentration, reabsorption effects were avoided. For more details see [2].

\section{RESULTS CONCERNING ENERGY LEVELS OF THE SUBSTITUTED POLYENE MOLECULES}

\subsection{INDIVIDUAL COMPONENTS}

Fig. 2 shows the absorption spectra of anthracene, naphthalene, TPP and the polyene $P_{9}$, in $n$-hexane, for comparison with the spectra of the more complicated molecules which will be discussed in the following. One can easily identify the well known lower singlet transitions $s_{1}$ to $s_{3}$ of $N$ and $A[3]$, the so called $Q$ and the Soret band of TPP [4], and the strong singlet $S_{2}$-absorption of $P_{9}$ with some vibronic structure. The spectra of other polyenes change in the well known way: with increasing number of double bonds, the long wavelength absorption shifts to longer wavelength and the extinction coefficient increases. [6] 


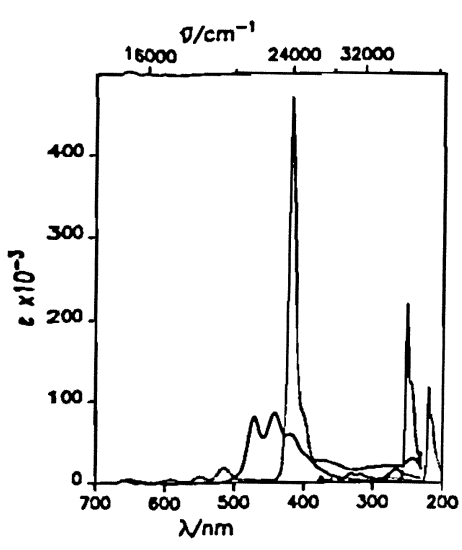

Fig. 2: Absorption spectra of $\mathrm{Pg}(\longrightarrow)$, TPP(----), $A(-\cdot-\cdot)$ and $N(\cdot \cdot)$ in $\mathrm{CH}_{2} \mathrm{Cl}_{2}$ at room temperature

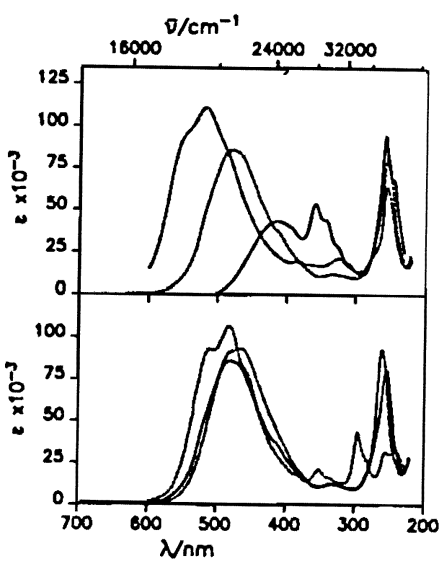

Fig. 3: a) (top) : Absorption spectra of $9 A-P_{n}-\mathrm{CHO} ; n=4(-), n=8(---)$, $n=12(-\cdots)$.

b) (bottom): Absorption spectra of $9 \mathrm{~A}-\mathrm{P}_{8}-\mathrm{CHO}\left(\longrightarrow, 2 \mathrm{~A}-\mathrm{P}_{8}-\mathrm{CHO}\right.$ $(--)$ anc 1,4 DM9A-P $8-$ CHO $(-\cdots)$

\subsection{POLYENES WITH ANTHRYL GROUPS AS SUBSTITUENT}

Fig. 3a shows the absorption spectra of $d_{1} f$ ferent polyenes $9 A-P_{n}-C H O(n=4,8,12)$, substituted at one end with $A$ in the 9-position. The polyene absorption (above $400 \mathrm{~nm}$ ) has less vibronic structure than the absorption of the unsubstituted polyenes (see F1g. 2). The $S_{3}$-absorption of $A(254-258 \mathrm{~nm}$ ) is shifted to lower energy as compared to Fig. 2, and the extinction coefficient is decreased by 558 . The $S_{1}$ absorption of $A(300-400 \mathrm{~nm})$ can not be identif led in detail.

The absorption spectra of $2 \mathrm{~A}-\mathrm{P}_{\mathrm{n}}-\mathrm{CHO}$, this means the anthryl group is connected to the polyenes in 2-position, (Fig. 3b) differs from the $9 A-P_{n}$-CHO spectra significantly: the polyene absorption is redshifted and more intense, the anthryl absorption at $250 \mathrm{~nm}$ is reduced, there is an additional band at $290 \mathrm{~nm}$, and the absorption between 300 and $400 \mathrm{~nm}$ wh1ch is due to the $s_{1}$-absorption of A is also changed.

The differences between the spectra shown in $F i g .3 a$ and $3 b$ show already that the amalgamation between the polyene levels and those of the substituent varies depending on the length of the polyene and on the position in which the substituent is linked to the polyene.

This is further demonstrated in Fig. 3b with 1.4 dimethylanthracene (1.4 DMA) as substituent. The absorption in the $A$ region is decreased in intensity as compared to $1.4 \mathrm{DMA}$ in solution, and similar to the A-absorption with $9 \mathrm{~A}$ as substituent. The polyene absorption is also very similar to that of the compounds $9 A-P_{n}-C H O$. 
Substitution of $A$ at both ends of the polyene results in an absorption spectrum which is almost identical to the single substituted molecule except a factor of the order two in the extinction coefficient in the A region of the spectrum (Fig. 4).

Two examples of emission spectra of molecules $9 \mathrm{~A}-\mathrm{P}_{\mathrm{n}}-9 \mathrm{~A}$ are also given in Fig. 4 . The emission spectra are superpositions of polyene emission in the long wavelength part of the spectrum and A emission at shorter wavelength. These two parts of the emission spectrum can be identified easily both by their vibronic structure and by comparison with the spectra of the molecules $A$ and polyene alone. The relative intensity of both parts of the emission spectra is excitation dependent. For instance in $9 A-P_{5}-9 A$, Fig. $4 a$, the polyene emission shows up when exciting in the polyene absorption $(400-500 \mathrm{~nm})$ or at $313 \mathrm{~nm}$. The A emission is strongest when exciting at $257 \mathrm{~nm}$, that is within the $s_{3}$-absorption of $A$.

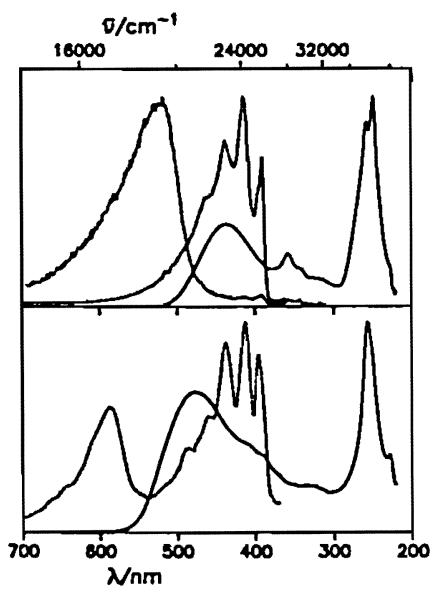

Fig. 4: a) (top): Spectra of $9 A-P_{5}-9 A$. $A b-$ sorption $(\longrightarrow$ and emission spectra (after excitation at $313 \mathrm{~nm}$ : ----; at $257 \mathrm{~nm}:-\cdot-\cdot)$

b) (bottom) : Spectra of $9 A-\mathrm{P}_{9}-9 \mathrm{~A}$. Absorption (-) and emission spectra (--) after excitation at $361 \mathrm{~nm}$. All emission spectra are normalized on the same maximum intensity.

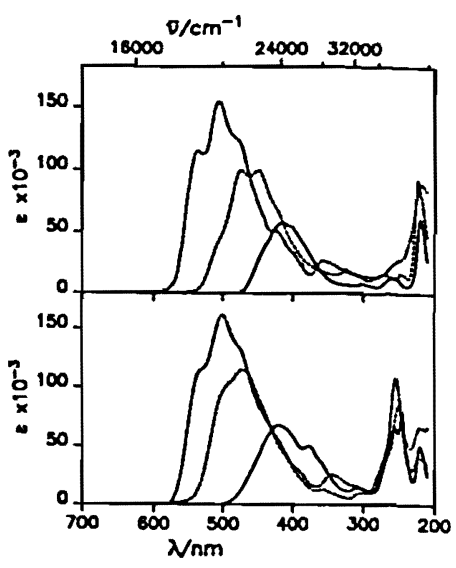

Fig. 5: Comparison of the absorption spectra of $1 \mathrm{~N}-\mathrm{P}_{\mathrm{n}}-1 \mathrm{~N}$ (top) and of $1 \mathrm{~N}-\mathrm{P}_{\mathrm{n}}-9 \mathrm{~A}$ (bottom) $n=5 \longmapsto), n=9(---), n=13(-.-)$ (solution in $n$-hexane)

\subsection{POLYENES WITH NAPHTHYL GROUPS AS SUBSTITUENTS}

When naphthyl groups instead of anthryl groups are substituted (Fig. 5a), the polyene part of the absorption spectrum is similar to the anthryl compounds. In the short wavelength part of the spectrum, the $A$ absorption is replaced by absorption bands between 250 and $200 \mathrm{~nm}$ which can be identified as naphthyl absorption by comparison with the naphthalene absorption spectrum, Fig. 2. Absorption structures in the region between 250 and $350 \mathrm{~nm}$ are apparently due to the lower Sstates of naphthyl, but the identification is not so clear. 
Finally, when having both $N$ and $A$ as substituent, the absorption spectra of the molecules $\left(1 N-P_{n}-9 A\right.$ in Fig. $\left.5 b\right)$ are superpositions of the $P_{n}, N$ and $A$ absorption as identified in the previous spectra.

\section{4. SUMMARY AND DISCUSSION}

The spectra of substituted polyene molecules show clearly, that the different partners which are linked into one large molecule keep their identity to a high degree as far as electronic levels and vibronic structure is concerned. A selective excitation of the aromatic end groups is possible. There is no complete amalgama$t$ ion of the energy levels into new levels of the "supermolecule".

On the other hand, there is clear evidence for characteristic changes in the molecular levels of the partners when being bound to the supermolecule. Such changes are

- Energy shift and loss of vibronic structure in the polyene absorption

- Decrease of extinction coefficient, line broadening and energy shift in the $S_{3}$ region of the $\mathrm{N}$ and $A$ absorption

- Change in structure and energy of absorption which can not be identified in all detall in the spectral region where $A$ and $N$ absorptions are weaker, this is between 300 and $380 \mathrm{~nm}$.

Furthermore, these characteristic changes of the spectra are dependent not only on polyene length but also on orientation of the substituent relative to the polyene chain. Anthracene substituted in its 2 position is more strongly influenced than in its 9 position. In molecules with long polyene chains, the changes are less pronounced than in the molecules $P_{4}$ and $P_{5}$. This is especially evident for the new strong line in the $A$ region of the spectrum of $9 A-P_{4}-C H O, F i g .3 a$.

These observations seem to indicate that the degree of amalgamation of the electronic levels of the substituents with those of the polyene chain is strongest when the chain is short, and when the steric situation of the substituent does not prevent a planar alignment between the polyene chain and the plane of the aromatic substituent. Anthracene substituted in 9-position can not be arranged coplanar with the polyene chain, in contrast to the 2-substitution. The spectra with 1.4-DimethylAnthracene as substituent are similar to those of 9-A, in agreement with the noncoplanarity due to steric hindrance.

\section{RESULTS CONCERNING INTRAMOLECULAR ENERGY TRANSFER}

\section{1. SPECTRA OF TPP SUBSTITUTED POLYENES}

In order to find out how energy can be transferred from one end of the molecule (donor) to the other end (acceptor), we used the molecules $9 A-P_{n}-T P P$ with the anthryl group which absorbs below $370 \mathrm{~nm}$ and emits between 370 and $450 \mathrm{~nm}$ as donor and TPP as acceptor. TPP absorbs strongly around $400 \mathrm{~nm}$ and weakly between 500 and $650 \mathrm{~nm}$ (see Fig. 2). It emits below $650 \mathrm{~nm}$. So the emission region of both end groups can clearly be separated. This is important for identifying 
a possible energy transfer.

Fig. 6a shows the absorption spectrum of polyenes substituted with TPP at one end only. In the region between 400 and $500 \mathrm{~nm}$, the absorption spectrum is dominated by the TPP soret band $(428 \mathrm{~nm})$. The polyene $\mathrm{s}_{2}$-absorption is superimposed with its maximum at $464 \mathrm{~nm}\left(P_{4}\right)$ and $507 \mathrm{~nm}\left(P_{8}\right)$. The structured absorption between 500 $\mathrm{nm}$ and $700 \mathrm{~nm}$ in the $\mathrm{P}_{4}$ spectrum and the broad absorption peak at $600 \mathrm{~nm}$ in the $\mathrm{P}_{8}$ spectrum are due to TPP. The shoulder at $357 \mathrm{~nm}$ is probably the $B_{y}$ component of the TPP soret band.

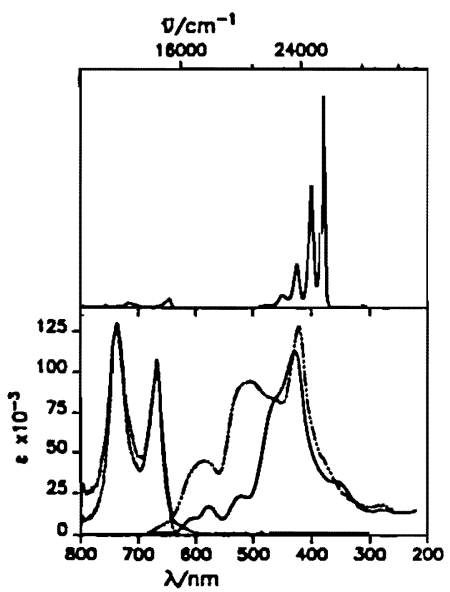

Fig. 6: a) (top): Emission spectrum of A and TPP (1:1) in solution, after excitation at $257 \mathrm{~nm}$. The spectrum corresponds to a superposition of the individual spectra.

b) (bottom) : Spectra of OHC-P - TPP Absorption ( $n=4:-, n=8:-\ldots)$ and emission spectra ( $n=4$, after excitation at $428 \mathrm{~nm}$ : ----, $n=8$, exc. at $257 \mathrm{~nm}:-\cdots \cdot)$

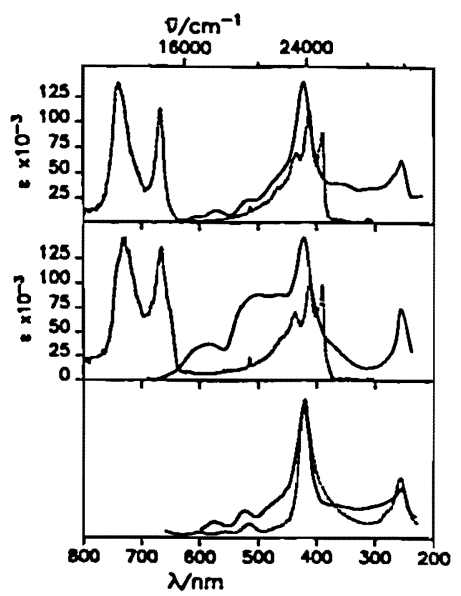

Fig. 7: a) (top): Spectra of 9A-P5-TPP. Absorption (-) and emission spectrum (---) after excitation at $257 \mathrm{~nm}$.

b) (middle) : Spectra of 9A-P9TPP. Absorption (-) and emission spectrum (---) after exc1tation at $257 \mathrm{~nm}$.

c) (bottom): Excitation spectra of $9 A-P_{n}-$ TPP $(n=5:-; n=9:-\cdots)$. Detection of the TPP-emission at $665 \mathrm{~nm}$.

The emission 1s, independent of the excitation wavelength, TPP fluorescence with two maxima at 667 and $738 \mathrm{~nm}$, Fig. 6b. Fig. 7 shows the absorption spectra of the polyenes $\mathrm{P}_{5}$ and $\mathrm{P}_{9}$ which are substituted at one end with TPP and at the other end with $A$. In addition to slightly shifted polyene and TPP absorption as compared to (Fig. 6b), one observes pronounced A absorption with a maximum at $256 \mathrm{~nm}$, characteristic for anthryl substitution.

The emission spectra of the twofold substituted polyene molecules $9 A-P_{n}-T P P$ depend on excitation wavelength. The emission with maxima at 667 and $738 \mathrm{~nm}$ is always present, when exciting into the TPP or the polyene or the A part of the ab- 
sorption spectrum. When the excitation wavelength is shorter than $360 \mathrm{~nm}$ (see Fig. 7), in addition the typical anthracene emission with origin at $390 \mathrm{~nm}$ is observed, red shifted by $820 \mathrm{~cm}^{-1}$ as compared to $A$ in $n$-hexan (Fig. 2). The A intensity is much weaker relative to the TPP intensity as compared to an emission spectrum of a solution in which the substituents A and TPP are dissolved separately with concentrations equal to those used for taking the spectra in Fig. 7, see Fig. 6a.

In order to identify further the energy transfer from the $A$ end to the TPP end of the supermolecules 9-P $\mathrm{P}-\mathrm{TPP}$, excitation spectra are very important. Flq. 7c shows as an example the intensity of TPP emission $(650$ to $750 \mathrm{~nm})$ as a function of the wavelength. Most important are the following results:

- The excitation spectrum is dominated by a pronounced maximum at $422 \mathrm{~nm}$ which is due to the $s_{2}$-absorption of TPP

- The second maximum at $255 \mathrm{~nm}$ belongs to the $S_{3}$-absorption of the $A$ group

- For the molecule $A-P_{5}-$ TPP, there is good agreement between the excitation spectrum and the absorption spectrum (Fig. 7a) also in the region between 600 and $420 \mathrm{~nm}$, that is the superposition of polyene and TPP absorption.

- For the molecule 9A-P 9 -TPP on the other hand, there is good agreement between the absorption spectrum (Fig. 7b) and the excitation spectrum only for excitation below $420 \mathrm{~nm}$. In the region between 650 and $420 \mathrm{~nm}$, the broad absorption maxima at 585 and $501 \mathrm{~nm}$, respectively, which have been sdentified as due to polyene, are absent. Instead, structures which obviously are due to the Q-bands of TPP ( $\left.\varepsilon_{1}-s t a t e\right)$ show up which can not be identified in the absorption spectrum due to superimposed polyene absorption.

\section{2. TIME RESOLVED MEASUREMENTS}

The decay constants after short (30 ps) pulse excitation of the A part $(415 \mathrm{~nm})$ and the TPP part $(655 \mathrm{~nm})$ in the emission spectra of the doubly substituted molecules $A-P_{5}-$ TPP and $A-P_{9}-$ TPP are within the error $11 \mathrm{mits}$ equal, $4.5 \pm 0.5$ ns. These are also the decay constants for the molecules $A$ and TPP in solution. So there 15 no decrease of the "donor" decay constant induced by energy transfer to the acceptor.

\section{3. DISCUSSION: ENERGY TRANSFER}

We believe that the experimental results can be understood only by assuming a mechanism of intramolecular energy transport within the molecule, using an electronic level higher than the lowest $S$ levels of the three constituents of the molecule and common to the whole supermolecule as vehicle for this transfer.

The existence of transfer of energy absorbed by the $A$ group and transferred to the TPP group follows from the emission and excitation spectra, Fig. 7. Since the decay constants are not changed by this energy transfer, the emitting states of both the donor and the acceptor must be lower in energy than the state which is responsible for energy transfer. 
In order to explain the observed energy transfer as an intramolecular mechanism, one has to exclude intermolecular transfer. Reabsorption can be excluded by the independence of the results from concentration in the whole range of concentrations measured $\left(10^{-5}-10^{-7} \mathrm{~mol} / 1\right)$. This is also true for Förster transfer. In addition, Förster transfer should result in a shortening of the donor emission which is not observed.

In the supermolecule $9 \mathrm{~A}-\mathrm{P}_{5}-\mathrm{TPP}$, there is obviously an energy transfer from the $S_{2}$ state of polyene to the emitting state of TPP (Fig. $\left.7 a, c\right)$ which could perhaps be explained as Förster transfer (similar to results in [5]).

In summary, we believe that our measurements give evidence for an intramolecular energy transfer in the molecules $9 A-P_{n}-T P P$. The energy which is absorbed by the anthryl substituent relaxes at least partially into an energy level which belongs to the whole supermolecule as result of amalgamation between the electronic states of the three constituent molecules. After further relaxation into the lowest excited S-state of the TPP group, TPP fluorescence emission is observed.

More detailed work especially on quantum yields and decay functions is in progress.

\section{ACKNOWLEDGEMENT}

Financial support by Deutsche Forschungsgemeinschaft, SFB 329, is gratefully acknowledged.

\section{REFERENCES}

1 F. Effenberger, H. Schlosser, P. Băuerle, St. Maier, H. Port, H.C. Wolf Angew, Chemie, 100 (1988) 274.

2 St. Maier, Dissertation, Universitát stuttgart (1988).

3 J.B. Birks, Organic Molecular Photophysics, 1 (1973), John Wiley \& Sons, London.

4 D. Dolphin (ed.), The Porphyrins, Academic Press, New York (1978).

5 G. Dirks, A.L. Moore, T.A. Moore, D. Gust, Photochem. Photobiol., 32 (1980) 277.

6 B. Hudson, B. Kohler, K. Schulten, Excited States, Vol. 6, E.C. Lim (ed.), Acad. Press, New York (1982). 